\title{
Tobacco Control in the South-East Asia Region: Accelerating Impact by Building on the Achievements and Navigating the Challenges
}

\author{
Vinayak Prasad ${ }^{1}$, Jagdish Kaur ${ }^{2 *}$
}

Asian Pac J Cancer Prev, 22, Progress of Tobacco Control in the South-East Asia Region Suppl, 3-5

Tobacco is one of the greatest threats to global health today. Reducing tobacco use is extremely critical to mitigating the burden of noncommunicable diseases (NCD), which account for $71 \%$ of the global deaths (WHO, 2021). It is a major public health concern in the South-East Asia (SEA) Region of the World Health Organization (WHO). In line with the Sustainable Development Goals, the Region is striving towards achieving a one-third reduction in premature deaths caused by NCD by 2030 . Effective tobacco control across the countries of the Region would significantly contribute to achieving this target. The Region is expected to achieve only a $6 \%-7 \%$ relative reduction in smoking rates among men between 2010 and 2025 and a relative reduction of around $70 \%$ among women during the same period (WHO, 2019). The rising trend of smokeless tobacco use among youth and women is particularly alarming in the Region. Among adult women, smokeless tobacco users outnumber the estimated number of smokers in the Region (WHO, 2019). Recent reports indicate increasing use of smokeless tobacco products and areca nut products by young individuals (WHO, 2020a; WHO, 2020b; and WHO, 2020c). In many countries, while the prevalence of smoking is decreasing, the use of smokeless tobacco is on the rise. Smokeless tobacco consumption has the potential of becoming a regional threat of massive proportion, based on its social acceptance, aggressive marketing strategy, attractive packaging, flavouring and affordability.

To address this growing public health malady, the countries of the Region have taken significant steps towards building enabling environments to promote tobacco-free cultures and lives. This editorial delves into some of the recent tobacco control initiatives of these countries and also highlights the key challenges in tobacco control faced by the Region. All the countries of the Region, except Indonesia, are Parties to the WHO Framework Convention on Tobacco Control (FCTC). Besides, all the countries are committed to implementing the MPOWER measures, which correspond to the articles of the Convention and were introduced by the WHO to assist the countries of the world in reducing the demand for tobacco products.

\section{Building on the Achievements}

The Region remains committed to monitoring of tobacco use prevalence data. The Tobacco Questions for Surveys (TQS) are being increasingly integrated in national health surveys or multiple risk factor surveys, as per country needs and context. The aim is to improve survey comparability over time and harmonize regional tobacco surveillance and monitoring activities. In the year 2000 , Region had total tobacco use rates at around $47 \%$. Notably, owing to sustained tobacco control efforts on part of the member countries, the Region is tracking towards an average prevalence rate of $25.1 \%$ in 2025 (WHO, 2019). Second round of Global Adult Tobacco Survey (GATS) has been completed in 2017 in Bangladesh and India, and shows sustained progress. GATS is currently underway in Indonesia and Sri Lanka. Recent Global Youth Tobacco Surveys (GYTS) have been accomplished in Bhutan, India, Indonesia, Maldives, and Timor-Leste. Bangladesh, Nepal, and Thailand are currently in the process of undertaking GYTS. As per Global Tobacco Control Report (GTCR) 2021, five countries of the Region-Bangladesh, Bhutan, Indonesia, Myanmar, and Thailand-have recent, representative and periodic tobacco prevalence data for both adults and youth (WHO, 2021).

All the countries are striving to effectively and holistically implement the smokefree laws. As per GTCR 2021, Nepal and Thailand have all public places completely smoke-free or at least $90 \%$ of the population covered by complete subnational smoke-free legislation. Additionally, Bhutan and DPR Korea need to cover only one more place (cafés, pubs \& bars and government facilities, respectively) with a smoking ban to join the exclusive list of countries with comprehensive smoke free laws (WHO, 2021). More and more countries of the SEA Region are implementing effective tobacco cessation programmes. India has strategically invested in scaling up tobacco cessation through mCessation services and 
the toll-free National Tobacco Quitline, which has four hubs providing multilingual services catering to different regions of the country (WHO, 2021).

The Region is doing exceptionally well in implementation of large graphic health warnings on tobacco packs, with countries like Bangladesh, India, Myanmar, Nepal, Sri Lanka, Thailand and Timor-Leste taking the lead. In fact, by the end of 2020, Thailand is among the 17 countries of the world, and first in Asia, to implement plain packaging of tobacco products (WHO, 2021). Maldives recently improved its legislation paving way for implementation of large and effective graphic health warnings. Indonesia, Myanmar, Thailand and Timor-Leste are conducting regular national anti-tobacco campaigns with at least seven appropriate characteristics including airing on television and/or radio (WHO, 2021). Maldives and Nepal have comprehensive bans on all forms of direct and indirect tobacco advertising or at least $90 \%$ of the population covered by subnational legislation completely banning tobacco advertising, promotion and sponsorship (TAPS). India needs only to ban advertising of tobacco products at "point of sale" to achieve the highest level in ban on TAPS. Sri Lanka and Thailand have been placed in the best-practice group by levying taxes that comprise at least $75 \%$ of retail prices of the most sold brand of cigarettes and need only to ban brand-stretching to achieve the highest level in TAPS ban (WHO, 2021).

Smokeless tobacco is banned in DPR Korea, India, Nepal, Sri Lanka, and Thailand (Kaur, Thamarangsi and Rinkoo, 2017). Electronic nicotine delivery systems (ENDS) are banned in DPR Korea, India, Nepal, Sri Lanka, Thailand, and Timor-Leste; these products are regulated in Maldives (WHO, 2020d). Additionally, DPR Korea, India, and Timor-Leste are among the 11 countries of the world that have completely banned (sales ban or another type of ban that restricts their availability) heated tobacco products (HTPs) (WHO, 2021). The Region contributes to the global tobacco control through FCTC Global Knowledge Hub on smokeless tobacco (India), Knowledge Hub to track tobacco industry interference in public policy-making (Thailand) and Centre for Combating Tobacco (CCT) (Sri Lanka tobacco observatory on tobacco industry interference). India has established three tobacco testing laboratories to support tobacco product regulation. The Regional Office for the SEA is supporting National Institute of Mental Health and Neurosciences (NIMHANS) Bengaluru, India to establish an additional tobacco testing lab to focus on testing of smokeless tobacco and flavors. All four labs are members of the WHO Tobacco Laboratory Network (TobLabNet) and supporting tobacco products regulatory activities at the regional and global level.

\section{Navigating the Challenges}

Despite the challenges of the COVID-19 pandemic, the Region has persisted in advancing tobacco control as a key health priority. Many of the achievements presented in the last section are testament to that perseverance. Despite policy interference by lobbying governments to reopen tobacco/cigarette factories early in the COVID-19 pandemic in countries such as Bangladesh and Indonesia, the tobacco control community showed ample resilience to continue with the good work. During COVID-19 lockdown in India, spitting bans (an act associated with chewing tobacco) were imposed in many parts of the country (Kaur and Rinkoo, 2020). Unfortunately, Bhutan, a country that has maintained a tobacco ban for over a decade, had to lift the ban to discourage cross-border transmission of COVID-19.

The progressive improvement in the quality of implementation of tobacco control measures has changed the dynamics of tobacco epidemic in the SEA Region. The big tobacco is increasingly targeting the implementation of tobacco control measures at the country level. New tobacco products have emerged aimed at renormalizing tobacco use, especially among youth. The smokeless tobacco industry is consolidating itself by enhancing its market. Gender-specific risks need to be increasingly addressed by all countries while developing tobacco control strategies (Kaur, Rinkoo and Prasad, 2017). Despite all challenges, it is encouraging to see that the countries of the Region are proactively gearing up to circumvent these constraints, especially in the context of new emerging products, such as ENDS and HTPs, and smokeless tobacco.

While sustaining demand reduction strategies, countries also need to focus on supply side issues addressing illicit trade in tobacco products, restricting access of tobacco products to minors and provisioning alternative livelihoods for tobacco growers and workers. As of now, in the SEA Region, only India and Sri Lanka are Parties to the Protocol to Eliminate Illicit Trade in Tobacco Products (WHO, 2020d). Remaining countries should consider becoming parties to the Protocol on priority. To counter targeting of youth by the resourceful tobacco industry, it is pertinent to diligently implement and enforce provisions related to restricting access of tobacco products, including new and emerging products, to children and youth in all countries. Tobacco growers and workers, often used by the industry as front groups to rally against tobacco control, are important stakeholders (Kaur, Rinkoo and Arora, 2014). The governments of tobacco growing economies, such as Bangladesh, India and Indonesia should establish mechanisms to support tobacco growers with economically viable alternative livelihood options and effective market support systems for successfully shifting over from cultivating tobacco.

\section{Accelerating Impact}

The path forward in context of effective tobacco control in the SEA Region is fraught with challenges, but this is the time to patiently and prudently build on what has been achieved so far. The desired way forward for the countries of the Region is the accelerated implementation of the WHO FCTC, including the supplyside interventions. A robust holistic approach that strictly firewalls interference in policy making by the tobacco industry, involves increased commitment and investment in tobacco control by the governments of the Region, and engages all other relevant stakeholders, including the civil society organizations, the international intergovernmental organizations, the UN organizations, and the academia 
will be a step forward towards the tobacco endgame in the SEA Region.

\section{Author Contribution Statement}

None.

\section{Acknowledgements}

The publishing cost was provided by WHO office in South-East Asia.

\section{Disclaimer}

The opinions or views expressed in this article are solely those of the author(s) and do not necessarily express the views or opinions of the organization to which the authors are affiliated.

\section{References}

Kaur J, Rinkoo AV, Arora S (2014). Operationalizing evidence into action for providing viable crop diversification options to tobacco farmers in India-a compelling case for change. Int J Interdiscip Multidiscip Res Stud, 2, 148-56

Kaur J, Rinkoo AV, Prasad VM (2017). Pattern and predictors of current smokeless tobacco use among women in selected states in India: Using the gender lens to interpret evidence from the global adult tobacco survey 2010. Glob J Med Public Health, 6, 1-6.

Kaur J, Thamarangsi T, Rinkoo AV (2017). Regulating smokeless tobacco and processed areca nut in South-East Asia region: The journey so far and the road ahead. Indian $J$ Public Health, 61, 3-6.

Kaur J, Rinkoo AV (2000). Public health perspectives of smokeless tobacco and Areca Nut use in the COVID-19 Era. Nicotine Tob Res, 22, 1660-61

World Health Organization (2019). WHO global report on trends in prevalence of tobacco use 2000-2025, third edition. Geneva: World Health Organization. Available from: https:// www.who.int/publications/i/item/who-global-report-ontrends-in-prevalence-of-tobacco-use-2000-2025-thirdedition (last accessed 7 July 2021).

World Health Organization (2020a). Global Youth Tobacco Survey (GYTS), Bhutan, 2019. New. Delhi, India: World Health Organization, Regional Office for South-East Asia. Available from: https://www.who.int/publications/i/ item/9789290227724 (last accessed 5 July 2021).

World Health Organization (2020b). Global Youth Tobacco Survey (GYTS), Maldives 2019. New Delhi, India: World Health Organization, Regional Office for South-East Asia. Available from: https://www.who.int/publications/i/ item/9789290228158 (last accessed 7 July 2021).

World Health Organization (2020c). Global Youth Tobacco Survey (GYTS), Timor-Leste 2019. New Delhi, India: World Health Organization, Regional Office for South-East Asia. Available from: https://www.who.int/publications/i/ item/9789290228394 (last accessed 15 July 2021).

World Health Organization (2020d). The Tobacco AtlasPerspectives from the South-East Asia Region. World Health Organization, Regional office for South-East Asia. Available from: https:/www.who.int/southeastasia/publicationsdetail/sear-tobacco-atlas (last accessed 21 July 2021).

World Health Organization (2021). WHO report on the global tobacco epidemic 2021: addressing new and emerging products. Geneva: World Health Organization.
Available from: https://www.who.int/publications/i/ item/9789240032095 (last accessed 15 July 2021).

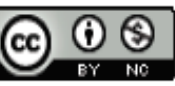

This work is licensed under a Creative Commons AttributionNon Commercial 4.0 International License. 